\title{
Anatomie - Eine historische Grundlage der Chinesischen Medizin und Akupunktur
}

\author{
Claus C. Schnorrenberger \\ Lifu International College of Chinese Medicine, Basel, Schweiz
}

$V$ iele, wenn nicht die meisten der historischen chinesischen Quellen der Akupunktur sind westlichen Akupunkteuren kaum bekannt.

Die „Bibel der Akupunktur“, der Nadel-Klassiker Huang-Di Nei-Jing Ling-Shu 黄帝內經霝樞, auch LingShu-Jing 靈樞經 genannt, berichtet im 12. Kapitel „Die pulsierenden Gefässe als Flussläufe“ (Jing-Shui 經水): " $\mathrm{Zu}$ messen wie hoch der Himmel und wie ausgedehnt die Erde ist, geht über menschliche Fähigkeiten hinaus. Es ist jedoch einfach, oberflächliche Messungen an einem Menschen auszuführen, der 8 Fuss lang ist. Nach seinem Tod kann sein Körper seziert werden, um einen allgemeinen Eindruck vom Erscheinungsbild, von der Grösse und vom Fassungsvermögen seiner Eingeweide zu gewinnen, die Länge seiner Blutgefässe $\mathrm{zu}$ bestimmen und die Menge und die Eigenschaften seines mit Sauerstoff (Atemluft) angereicherten zirkulierenden Blutes (Xue-Qi 血氣) abzuschätzen” [1].

Dieser Text, auf den wir noch zurückkommen werden, hat einen geschichtlich bezeugten Hintergrund. Wir finden das früheste chinesische Dokument einer Leichen-Öffnung in den Han Annalen 漢書. Hier wird in der Biographie des Kaisers Wang Mang 王莽 berichtet, dass dieser Herrscher im Jahre 16 unserer Zeitrechnung die Zergliederung des Körpers eines Rebellen namens Wang Sun-Ching anordnete, nachdem letzterer von der Armee gefangen genommen worden war. Die anatomische Sektion wurde durch den kaiserlichen Hofarzt Shang Fang und einen „geübten Metzger" durchgeführt [2].

Der Text erläutert das Verfahren: "Es wurden an der Leiche Messungen

Von den historischen Quellen der Akupunktur ist den westlichen Akupunkteuren vieles unbekannt. So sind die Namen der anatomischen Strukturen (innere Organe, Muskeln, Sehnen usw.), die in der modernen chinesischen Anatomie verwendet werden, dieselben, die schon im Buch Huang-Di Nei-Jing Ling-Shu 黃帝內經靈樞 überliefert werden. Auch die Schriftzeichen für die Technik der Anatomie sind noch dieselben, die in dem alten Text für die Leichensektion verwendet werden, nämlich Jie Pou 解剖. Wir finden das früheste chinesische Dokument einer Leichen-Öffnung in der Biographie des Kaisers Wang Mang 王莽 in den Han Annalen 漢書. Dieser Herrscher ordnete im Jahre $16 \mathrm{n}$. Chr. die ärztliche Zergliederung des Körpers eines Rebellen namens Wang Sun-Ching an. An Wangs Leiche wurden Messungen seiner inneren Organe vorgenommen, dünne Bambusruten wurden in die Blutgefässe eingeführt, um zu erkunden, wo sie beginnen und wo sie enden. Zahlreiche ähnliche anatomische Untersuchungen kamen im alten China hinzu und sind im Kapitel 12 des Huang-Di NeiJing Ling-Shu 黃帝內經靈樞, unter Jing-Shui 經水, beschrieben. Die untersuchten Gefässe sind die im Westen immer noch fälschlich als "Meridiane" bezeichneten Leitbahnen der Akupunktur. Darum sollte am Anfang jeder Akupunkturausbildung und vor jeder einschlägigen wissenschaftlichen Forschung das Studium einer anatomisch-wissenschaftlichen Nomenklatur für die mit den Nadeln durchstochenen Strukturen stehen, einschliesslich der mit innen gekoppelten inneren Organe, die auch nach 2000 Jahren noch die gleichen Gewebe sind wie die der modernen westlichen Heilkunde.

Schlüsselwörter: Klassische Akupunktur-Quellen, Anatomie im frühen China, moderne Organ-Namen in chinesischen Klassikern, anatomische Nomenklatur für Akupunkteure, sichere Stichtechnik, deutsche Gerac-Studie

\section{Anatomical Roots of Chinese Medicine and Acupuncture}

Western acupuncturists know little about the historic sources of acupuncture. The names of the anatomic structures (internal organs, muscles, tendons etc.) used in modern anatomy are similar to those mentioned in the book Huang-Di Nei-Jing Ling-Shu 黃帝內經靈樞. The same applies to the ancient Chinese characters for body dissection given here, namely Jie Pou 解剖. The earliest historic reference to a dissection of a human cadaver is found in the biography of Emperor Wang Mang 王莽 in the Han annals 漢書 who in 16 C.E. ordered the medical dissection of the body of a rebel named Wang Sun-Ching. Measurements were made of his internal organs, and bamboo rods were inserted into his blood vessels in order to discover where they begin and where they end for the purpose of a better understanding of how to cure diseases. Anatomical dissections are mentioned in chapter 12 of the Huang-Di Nei-Jing Ling-Shu 黃帝內經靈樞 entitled Jing-Shui 經水. The courses of the pathways laid out in chapter 10 of the Ling-Shu-Jing are basic for acupuncture and could well be the result of such dissections. The majority of Western acupuncturists still adhere to the assumption of "meridians". Therefore, modern acupuncture should finally be supplied with an anatomical nomenclature for daily practice and scientific research which incorporates all the structures involved which are still the same as some 2000 years ago.

Key Words: Classical sources of acupuncture, anatomy in ancient China, organ names in ancient Chinese books, anatomical nomenclature for acupuncturists, safe acupuncture techniques, Gerac German Acupuncture Trials

der inneren Organe vorgenommen, dünne Bambusruten wurden in die Blutgefässe eingeführt, um zu erkunden, wo diese Gefässe beginnen und wo sie enden. Dies soll dazu beitragen, ein besseres Verständnis für die Heilung von Krankheiten zu gewinnen [2].“

Da die Untersuchung von einem 


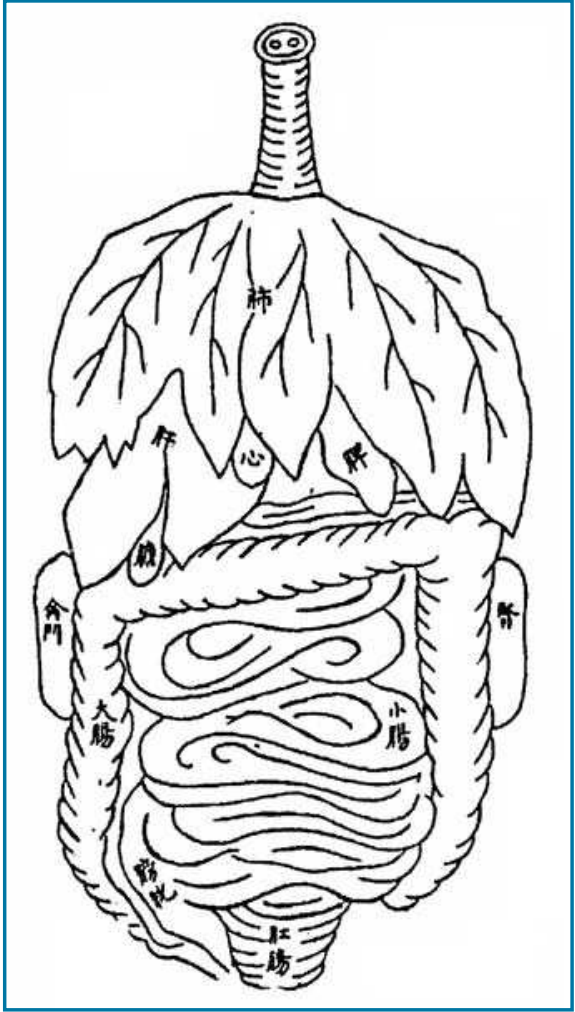

Abb. 1. Vorderansicht von Ou Xi-Fan's "Fünf Inneren Organen" 歐希范五臟圖. Sichtbar sind Trachea, Lunge, Herz, Milz, Leber, Gallenblase, linke und rechte (Ming-Men 命門) Niere, Dünndarm, Dickdarm und Mastdarm [6].

Arzt geleitet wurde, handelte es sich wohl um das erste anatomische Experiment in der Medizingeschichte überhaupt. Ein ähnliches Vorgehen wird im 12. Kapitel des Buches Ling-Shu-Jing geschildert. Ziel der Untersuchungen war, die Struktur der menschlichen Eingeweide, die Eigenschaften des Blutes und die Beschaffenheit der Blutgefässe zu erforschen. Offenbar war Kaiser Wang Mang, der in China bedeutende politische und ökonomische Reformen durchführte, sehr an Anatomie und am Fortschritt der Medizin interessiert. Er sammelte viele alte Schriften, zu denen vermutlich auch Texte gehörten, aus denen schliesslich das Werk Huang-Di Nei-Jing hervorging [1,3].

Auch in nachfolgenden Dynastien behauptete die Anatomie ihren Platz in der wissenschaftlichen Entwicklung von Chinas Heilkunde. Aus dem Buch Bin Tui Lu 䆩退錄 der Nördli-

Abb. 1-5: Einige Schriftzeichen für die gekennzeichneten Organe sind nicht klar erkennbar, was auf die dürftige Qualität des Originals zurückzuführen sein dürfte. chen Song-Dynastie (960-1127 n. Chr.) stammt folgender Bericht:

„In Kuang-Su wurde der Räuber $O u$ $X i$-Fan 歐希范 mit allen seinen Anhängern gefangen und getötet. Danach wurden im Verlauf von zwei Tagen 56 Personen anatomisch zergliedert. Der oberste Richter Wu Jian 吴簡 untersuchte die Körper sorgfältig, und er fertigte Zeichnungen davon an [4,5].“

Diese Massensektion wird mehrmals durch andere historische Quellen erwähnt. Die so entstandenen Illustrationen hiessen “Ou Xi-Fan's Bilder der Fünf Inneren Organe 歐希范五藏 圖” (Abb. 1) [6]. Sie wurden Jahrhunderte lang zur Ausbildung chinesischer Ärzte verwendet (Abb. 2, 3, 4, 5). Die Gefangenen wurden getötet, ihre Leiber geöffnet, Nieren und Eingeweide herausgeschnitten. Die Blutgefässe, Bänder, Sehnen, Muskeln und Organe wurden gesammelt und eingehend studiert. In seiner „Geschichte der Medizin“ (1964) erwähnt der japanische Medizinhistoriker TeIzo Ogawa den Abdruck von Ou Xi-Fan's Bildern

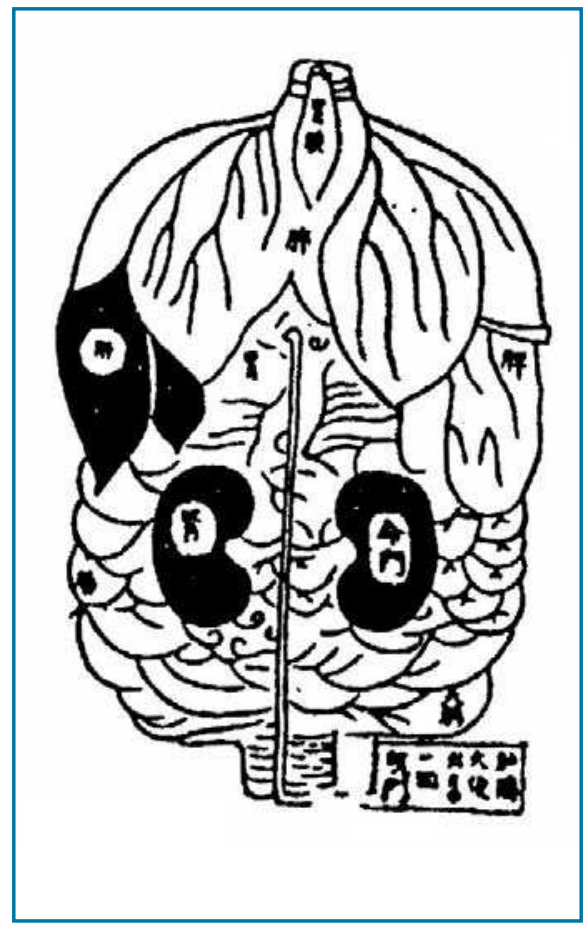

Abb. 2. Rückenansicht eines menschlichen Torso mit Lunge, Milz (geschwärzt links oben) Leber, Magen und Nieren (geschwärzt, Mitte) in einer chinesischen Darstellung aus der Nördlichen Song-Dynastie (10. Jahrhundert) [6] der Fünf Inneren Organe 歐希范五 藏圖 in dem alten japanischen Werk Ton-i-sho von Shozen Kajiwara, das zwischen 1302 und 1304 geschrieben wurde [5,7].

In Soochow wurden während der Song-Dynastie in der Chong-Ning 崇寧 Periode (1102-1106) zahlreiche Banditen hingerichtet. Mit Hilfe von Ärzten untersuchte der Präfekt Li Yi-Hang 李 夷行 deren innere Organe persönlich. Mediziner und Zeichner halfen ihm, davon eingehende Abbildungen anzufertigen. Die so entstandenen Bilder, die man für besser hielt als die älteren Bilder der Fünf Inneren Organe des $O u$ Xi-Fan, wurden von Yang-Jie 楊介 herausgegeben und „Atlas der Wahrheit“ Cun Zhen Tu 存貞圖 genannt. Auch sie dienten lange dem Studium chinesischer Ärzte.

Das Kapitel 31 des Huang-Di NeiJing Ling-Shu 黄帝內經靈樞 mit der Überschrift „Darm und Magen (Chang Wei 腸胃)“ enthält die Abmessungen und Gewichte der folgenden menschlichen Körperstrukturen [8]:

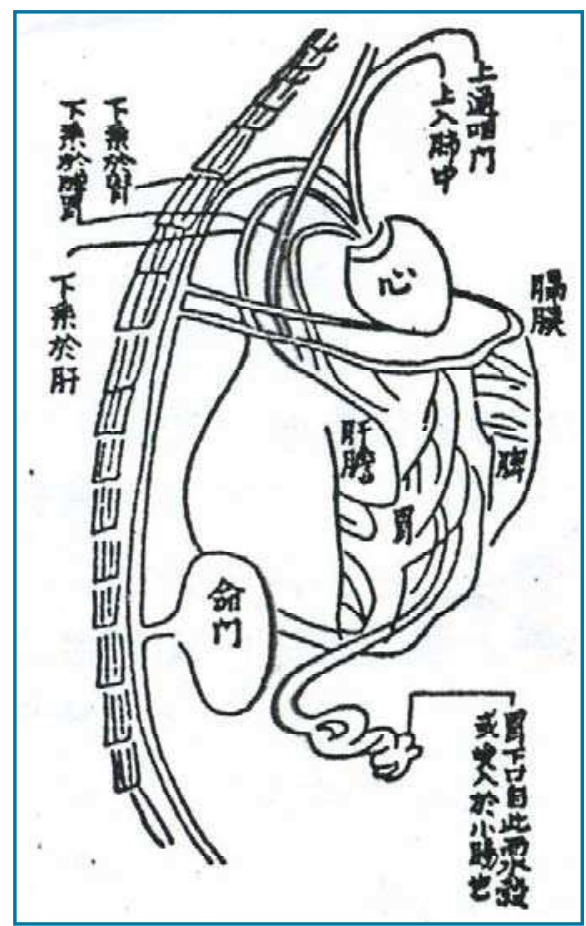

Abb. 3. Aus dem Buch "Wan An Fang" 萬安 方 (Zehntausend Hilfreiche Rezepturen), erschienen 1315: Dargestellt ist das Herz, das Zwerchfell, die Milz, die Leber, der Magen, die rechte Niere (Ming-Men 命門) und die vom Herzen zu den inneren Organen führenden grossen Blutgefässe des menschlichen Organismus [6]. 


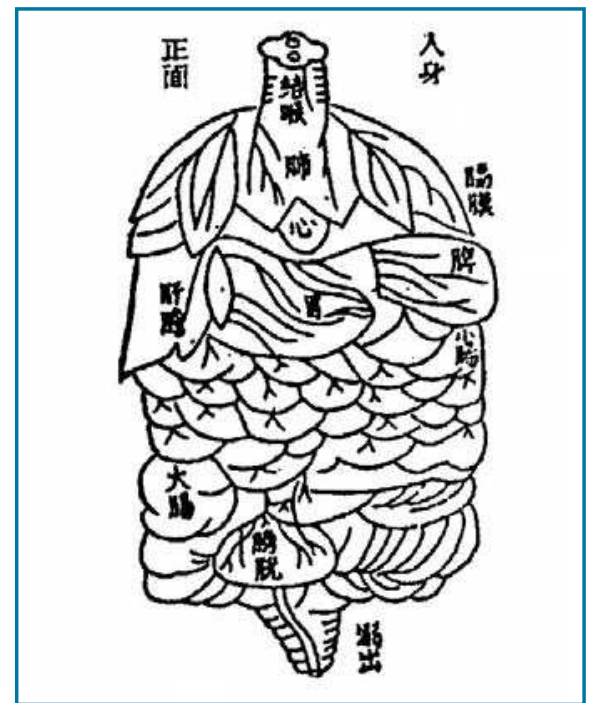

Abb. 4. Illustration aus dem Buch "Wan An Fang" 萬安方 (Zehntausend Hilfreiche Rezepturen) erschienen 1315: Vorderansicht der menschlichen Eingeweide mit Lunge, Herz, Milz, Leber und Gallenblase, Magen, Dünndarm, Dickdarm, Harnblase und Rektum (Mastdarm) [6].

Tab. 1. Historische chinesische Abmessungen der Darmabschnitte (aus Kapitel 31 des HuangDi Nei-Jing Ling-Shu 黃帝內經靈樞 [8]

\begin{tabular}{|lc|}
\hline $\begin{array}{l}\text { 1. Pharynx bis Mageneingang (Ösophagus) } \\
\text { 2. "Kleindarm” (Duodenum und Jejunum) }\end{array}$ & 1,6 chin. Fuss $=$ ca. $0,25 \mathrm{~m}$ \\
\hline $\begin{array}{l}\text { 3. "Krummdarm“ (Ileum mit Colon ascendens und } \\
\text { traversum) }\end{array}$ & 21 chin. Fuss $=$ ca. $3,60 \mathrm{~m}$ \\
\hline $\begin{array}{l}\text { 4. ., Breitdarm” (Colon descendens, Sigmoid und Rektum) } \\
\text { Ein chinesischer Fuss (Längenmass) entspricht ca. } 0,13-0,17 \mathrm{~m}\end{array}$ & 2,8 chin. Fuss $=$ ca. $0,37 \mathrm{~m}$ \\
\hline
\end{tabular}

Tab. 2. Vergleich der alten chinesischen mit den modernen anatomischen Massen

\begin{tabular}{lll} 
Dokumentationsquelle & $\begin{array}{l}\text { Längenverhältnis } \\
\text { Ösophagus : Intestinum }\end{array}$ \\
\hline Huang-Di Nei-Jing Ling-Shu & 1,6 chin. Fuss : 56,8 chin. Fuss $=1: 36$ \\
\hline Moderne Anatomie & $25 \mathrm{~cm}: 925 \mathrm{~cm}$ & $=1: 37$ \\
\hline
\end{tabular}

- Abstand der Lippen von der Zunge

- Breite des menschlichen Munds

- Abstand der Zähne von den Stimmbändern (Kehlkopf)

- Rauminhalt des Mundes

- Gewicht der Zunge

- Länge der Zunge

- Breite der Zunge

- Gewicht des Kehlkopfs

- Breite der Speiseröhre (des Schlunds)

- Länge der Speiseröhre

- Länge des Magens

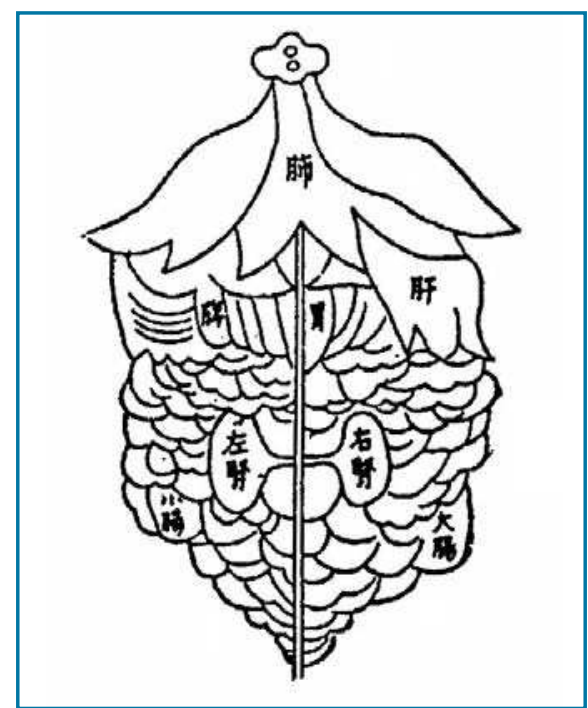

Abb. 5. Rückenansicht der inneren Organe mit Lunge, Leber, Milz, Magen, linker und rechter Niere, Dünndarm und Dickdarm. Abbildung aus dem Buch "Wan An Fang" 萬安方 (Zehntausend Hilfreiche Rezepturen) erschienen $1315[6]$

\section{Die Nomenklatur der modernen chinesischen Anatomie}

Die Namen der anatomischen Strukturen (innere Organe, Muskeln, Sehnen usw.), die von der modernen chinesischen Anatomie verwendet werden, sind noch dieselben, die im Buch $\mathrm{Hu}$ ang-Di Nei-Jing Ling-Shu 黄帝內經 靈樞 verwendet werden.

In seinem 12. Kapitel Jing Shu 經水 („Die Meridiane als Flussläufe“) werden die folgenden 11 inneren Organe aufgezählt: Harnblase, Gallenblase, Magen, Milz, Niere, Leber, Dünndarm, Dickdarm, Lunge, Herz, Perikard (Herzbeutel). Ferner sind die so genannten Drei Erwärmer (Dreiteiliger Erwärmer) San Jiao 三焦 erwähnt, welche die drei Ebenen des Rumpfes (Brustraum, Oberbauch, Unterbauch) topographisch repräsentieren und die einzelnen Organe zu einer Einheit zusammenfassen - ein Beispiel für die grundlegende Einheit des menschlichen Organismus [9].

Hier werden die Gefässverbindungen („Wasserwege“) von und zu 11 inneren Organen beschrieben, von denen die als „Flüsse“ bezeichneten pulsierenden Blutgefässe im Körper (in falscher westlicher Übersetzung „Meridiane“ genannt) entweder ausgehen oder zu denen sie hinführen (vgl. Abb. 3). Die verwendeten Schriftzeichen sind auf den beiden Originalseiten in Abbildung 6 leicht erkennbar:

膀胱 Pang Guang (Harnblase)

膽 Dan (Gallenblase)

- Durchmesser des Magens

- Fassungsvermögen des Magens

- Länge von Duodenum (Zwölffingerdarm) und Jejunum

- Umfang des Dünndarms

- Durchmesser des Dünndarms

- Topographie des Verlaufs vom Dickdarm (Kolon)

- Umfang des absteigenden Kolons

- Durchmesser des absteigenden Kolons
胃 Wei (Magen)

脾 Pi (Milz)

腎 Shen (Niere)

肝 Gan (Leber)

小腸 Xiao Chang (Dünndarm)

大腸 Da Chang (Dickdarm)

肺 Fei (Lunge)

心 Xin (Herz)

心包 Xin Bao (Perikard) 
Diese Schriftzeichen sind, wie bereits erwähnt, dieselben, die auch in der modernen chinesischen Anatomie verwendet werden.

\section{Anatomische Forschungen im alten China}

Die zentrale Stellung der Anatomie in Chinas Heilkunde, vor allem für die Akupunktur, wurde im Anfangszitat erwähnt. Diese Passage aus dem 12. Kapitel des Huang-Di Nei-Jing Ling$S h u$ 黄帝內經靈樞, „Die Meridiane als Flussläufe“ Jing-Shui 經水, weist unmissverständlich auf die Bedeutung der Anatomie nicht nur für die Akupunktur hin, sondern für die gesamte chinesische Heilkunde. Der kaiserliche Leibarzt Qi Bo 歧伯 erklärt darin dem
Kaiser Huang-Di 黄帝 die Technik zur Erforschung der grundlegenden Strukturen des menschlichen Organismus. Er sagt: Qi Si Ke Jie Pou 其死可解 剖. Übersetzung: “Wenn jemand gestorben ist, kann man ihn mit Hilfe der Anatomie (für das ärztliche Verständnis) zergliedern" [9].

Es ist interessant, dass die dabei verwendeten Schriftzeichen dieselben sind, die noch im modernen Chinesisch für das medizinische Fach der Anatomie verwendet werden, nämlich Jie Pou 解剖. Dies zeigt uns, dass die zeitgenössische Anatomie des heutigen China ursprünglich auf diesen Text des Ling-Shu-Jing zurückgeht. Bezeichnenderweise trägt ein modernes chinesisches Handbuch der Anatomie den Titel Tu Jie Ren Ti Jie Pou Xue Shou Ce 圖解人體解剖學手冊, d.h. Illus- triertes Handbuch der Anatomie des Menschen [10], und ein auf dem chinesischen Festland verbreitetes anatomisches Lehrbuch heisst Ren-Ti Jie-Po Xue 人体解剖學, „Lehrbuch der Anatomie des menschlichen Körpers“" [11].

Qi Bo erläutert an der zitierten Stelle, dass das Körperinnere eines Menschen durch Abmessungen an den oberflächlichen Strukturen der Körperaussenseite (Haut, Muskeln, Knochen u.a.) beurteilt werden kann, und dass seine inneren Organe, Zang Fu 藏腑, ihr Fassungsvermögen, ihr Inhalt, die Länge der Blutgefässe des Körpers, die Qualität des arteriellen und venösen Blutes durch die anatomische Zergliederung einer Leiche bestimmt werden können. Nach authentischer moderner Auffassung sind die Verläufe der Blutgefässe und Nervenbahnen, wie sie

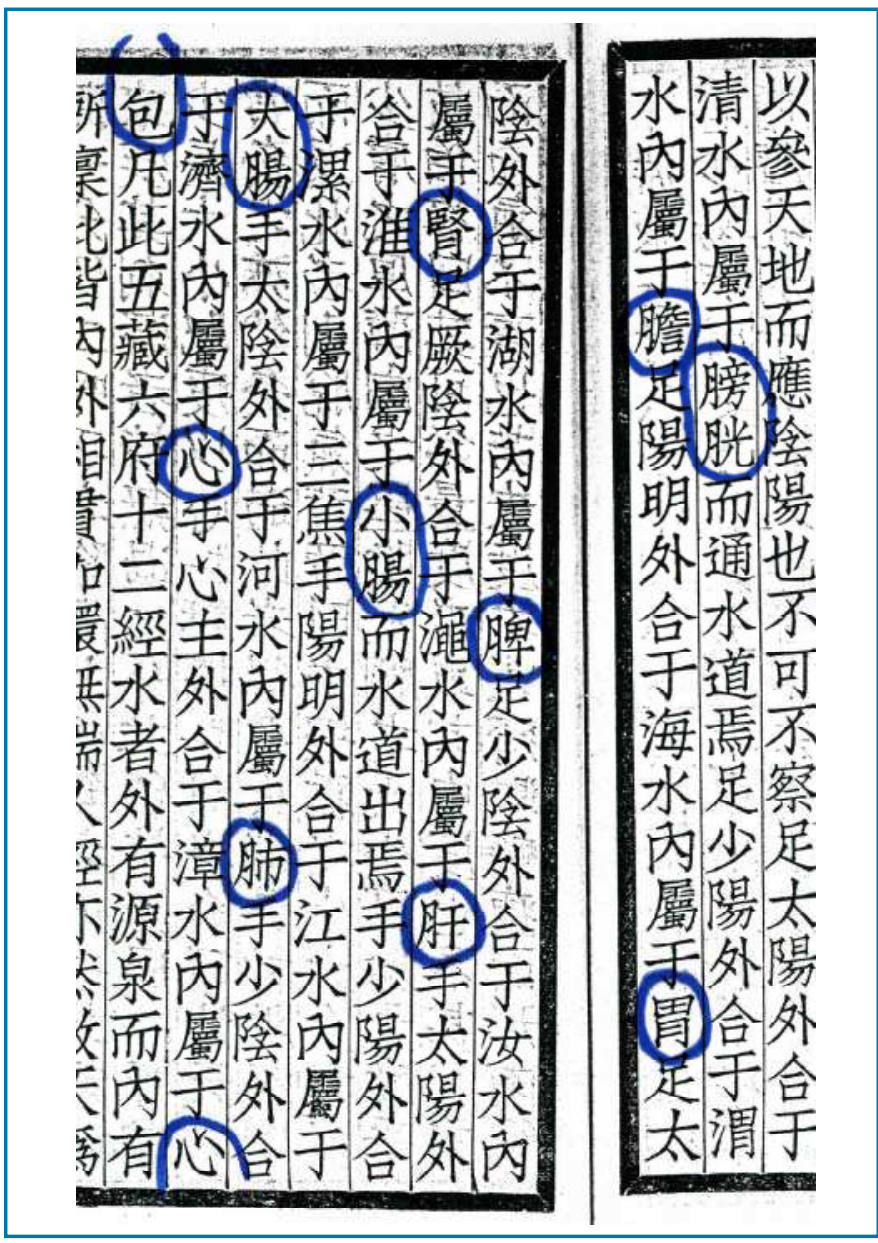

Abb. 6. Zwei Seiten aus dem Kapitel 12 (Jing-Shui 經水) des Huang-Di Nei-Jing Ling-Shu 黃帝內經靈樞 (vertikal zu lesen von rechts oben nach links unten) [9].

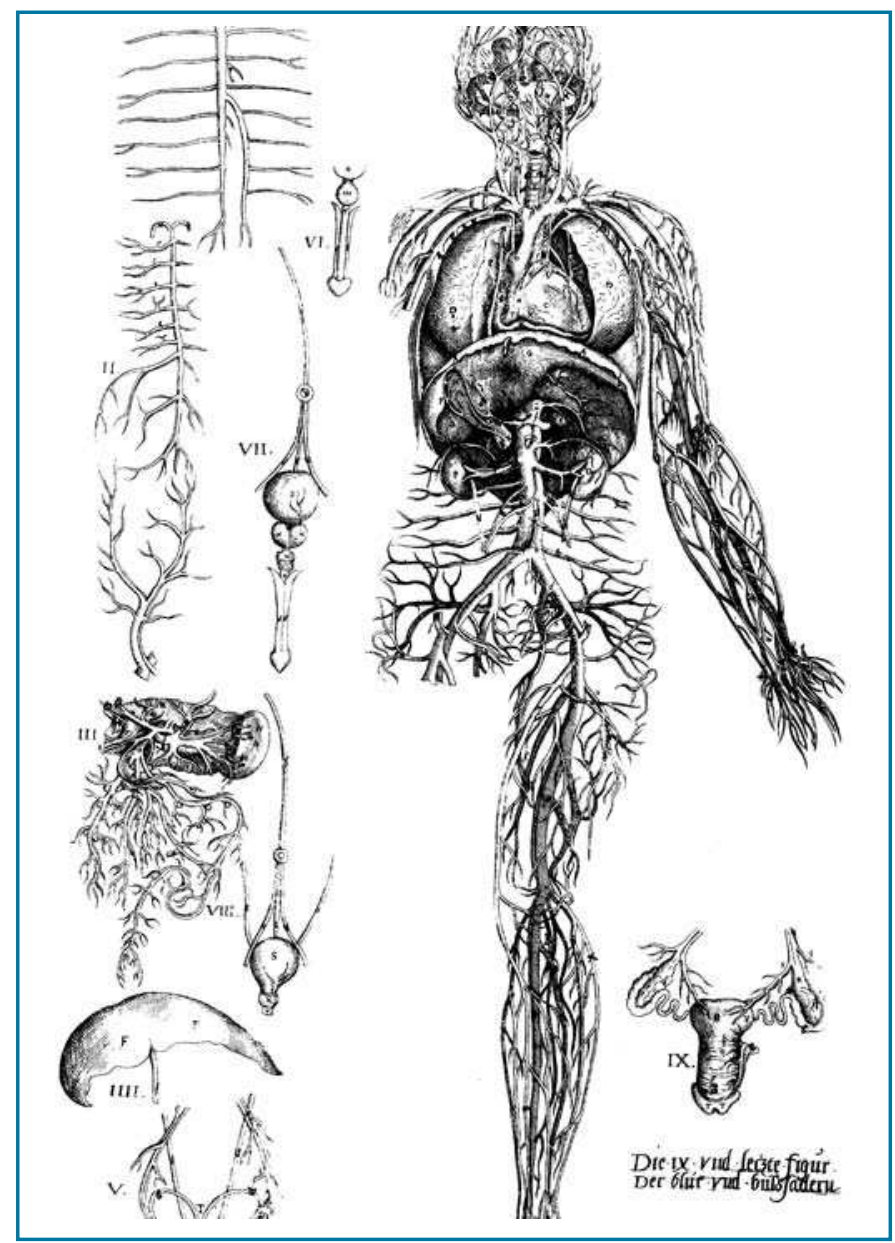

Abb. 7. Eine Abbildung aus Andreas Vesalius' „De Humani Corporis Fabrica", Basel, 1543 [14]. Das Zwerchfell mit hindurch tretenden Blutgefässen und darüber und darunter liegenden inneren Organen. Die dargestellte Topographie entspricht etwa der Beschreibung des Kapitels 10 im Ling-Shu-Jing [1]. 


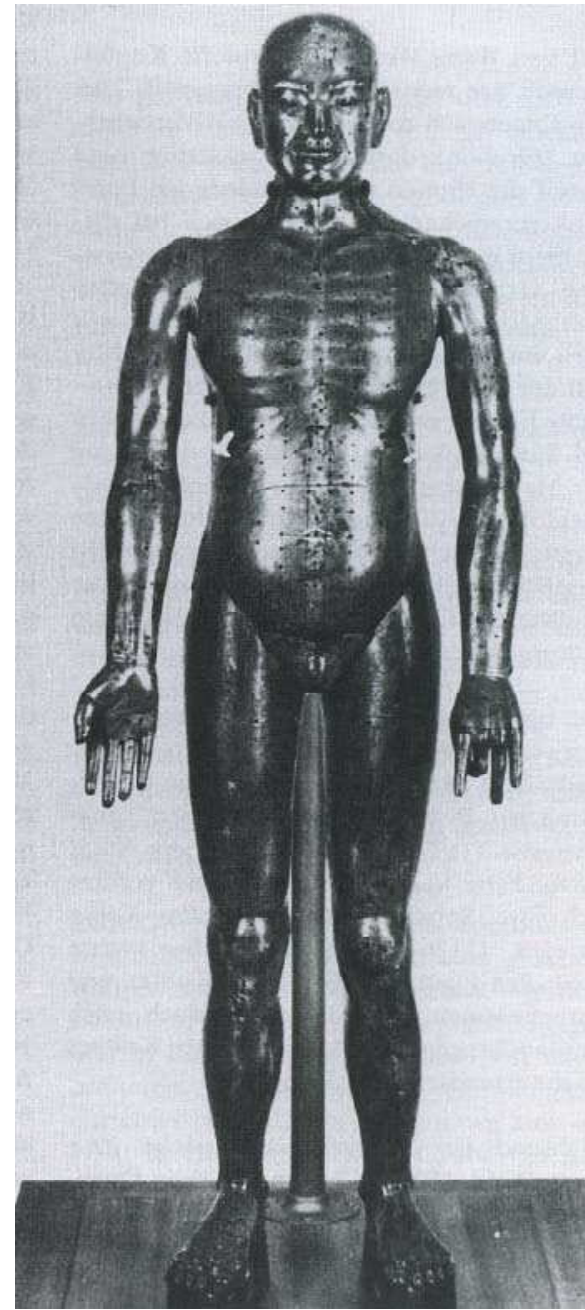

Abb. 8. Dieses Exemplar des originalen Kupfer-Menschen (Tong-Ren 銅人) befindet sich heute im kaiserlichen Museum in Tokio unter Verschluss. Seine anatomischen Masse entsprechen dem Körper eines heutigen Menschen. Die sichtbaren "Punkte” (chin. Xue-Wei 穴位) sind Löcher in den Platten aus Kupferblech, lateinisch Foramina $[15,19]$.

im Kapitel 10 des Ling-Shu beschrieben werden, letztlich das Ergebnis solcher anatomischen Zergliederungen $[12,13]$. Anders wäre unverständlich, wie die frühen chinesischen Mediziner 1. die einzelnen Organe überhaupt unterscheiden,

2. sie richtig benennen konnten,

3. ihre topographische Lage zuverlässig bestimmen konnten;

4. diese Organe dem das Rumpfesinnere teilenden Zwerchfell zuordnen konnten. (Das Zwerchfell ist nur nach der anatomischen Öffnung der Leibeshöhle sichtbar ist, was die Abbildung 7 aus Andreas Vesalius' Werk „De Humani Corporis Fabrica“ verdeutlicht) [14], und schliesslich

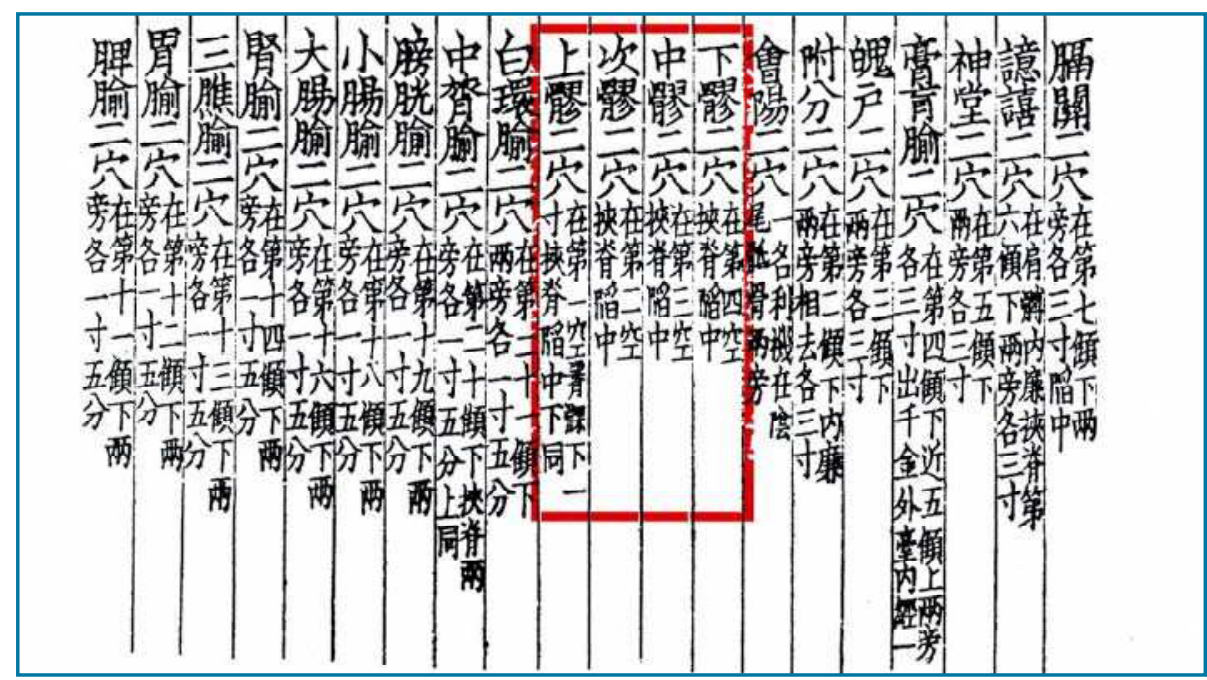

Abb. 9. Eine Seite aus dem Text des Wang Wei-Yi zum Kupfer-Menschen (Tong-Ren 銅人) mit der Beschreibung der Topographie der 8 Foramina sacralia (Ba Liao 八髎). [17] Die Bedeutung des Piktogramms Liao 㖒 ist "Knochenloch". In der westlichen Akupunktur-Terminologie werden die Ba Liao 八髎 indessen als „Punkte“ Blase 31, 32, 33 und 34 wiedergegeben. Letzteres ist eine Bezeichnung, die weder mit den his-torischen Quellen der Akupunktur noch mit der modernen Anatomie übereinstimmt $[19,20]$.

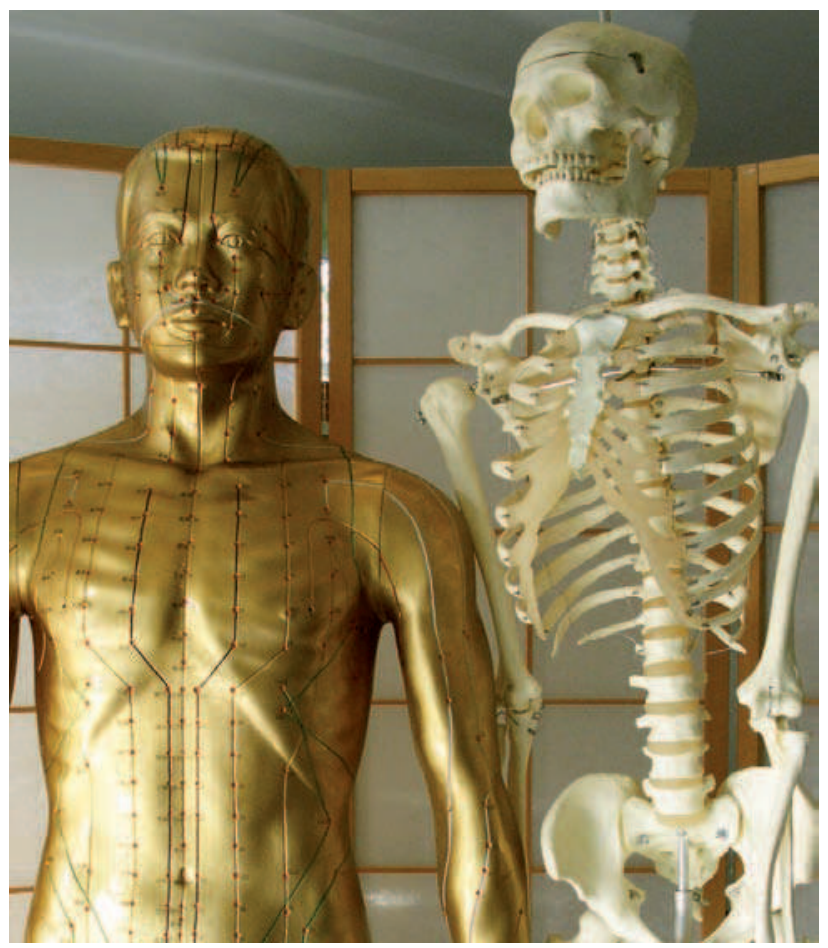

Abb. 10. Moderne taiwanesische Nachbildung des Kupfermenschen (links) von Wang Wei-Yi (1026) neben einem Skelett nach der westlichen Anatomie (rechts) [16].

5. die Blutgefässe, die zur Peripherie und ins Innere des Körpers führen, mit den einzelnen Organen in Verbindung bringen konnten.

Die Bahnenverläufe der chinesischen Heilkunde werden in der westlichen Akupunktur bis heute irrtümlich „Meridiane“ genannt, ein Terminus, der aus der Geographie stammt, wo er die Mittagslinien d.h. die Längenkreise der Erdkugel bedeutet, was mit den anatomischen und physiologischen Gegebenheiten des menschlichen Organismus nicht das Geringste zu tun hat. Die geographischen Meridiane, also die Mittagslinien am Globus, heissen auf Chinesisch $Z i-W u$-Xian 子午綫 (线)* oder 
auch Jing-Xian 經綫 (经线)*, wobei das Binom Jing-Xian 經綫 eine Linie bedeutet, die Mittagslinie der Erdkugel, weil 綫 Xian Linie heisst. Das ist etwas anderes als das 脉 Mai in 經脉 Jing Mai, den Gefässverläufen der Akupunktur, in welchen gemäss Kapitel 10 des Buches Ling-Shu-Jing das Xue-Qi 血氣 (血气)* d.h. das arterialisierte Blut ohne Anfang und Ende zirkuliert, womit das altchinesische Verständnis für den Blutkreislauf angedeutet ist.

\section{Anatomie im historischen China und im Westen}

\section{China}

Im Jahre 1026 konstruierte der kaiserliche Arzt Wang Wei-Yi 王惟auf Veranlassung des regierenden Song-Kaisers den berühmten KupferMenschen (Tong-Ren 銅人)** in zwei identischen Exemplaren. Die Figuren dienten der Ausbildung von Akupunkturärzten sowie für ihr Examen in zuverlässiger Stichtechnik, Topographie und den Strukturen des menschlichen Organismus. Es waren die ältesten Modelle des menschlichen Körpers in der Medizingeschichte überhaupt, die zu Unterrichtszwecken verwendet wurden. Ihre Abmessungen und Proportionen entsprachen dem Körperbau eines gegenwärtigen Menschen; ihre Strukturen waren also im modernen Sinne anatomisch korrekt dargestellt. Dies geht aus einer wissenschaftlichen Studie am einzigen erhaltenen Exemplar vom Kupfer-Menschen hervor, das sich heute im kaiserlichen Museum von Tokio unter Verschluss befindet [15]. Der Neuroanatom der China Medical University in Taichung/Taiwan, Professor Hongchien Ha M.D., hat diese Skulptur in den 80er Jahren untersucht und dabei seine völlige anatomische Korrektheit festgestellt. WANG WEI-YI publizierte zusammen mit seinen Figuren ein Lehrbuch über die topographischen und anatomischen Eigenschaften der Gefässverläufe und der Foramina („Punkte“), in die bei der Akupunktur Nadeln eingestochen werden (Abb. 8,

\footnotetext{
* maoistische Kurzzeichen der heutigen VR China in Klammern

** manchmal auch „Bronze-Mensch“ genannt
}

9, 10) [16]. Sein Text zum Tong-Ren wurde seinerzeit in Steinplatten gemeisselt, die in den frühen 70er Jahren beim Bau der Untergrundbahn in Peking in der Nähe des alten Kaiserpalastes teilweise wieder zum Vorschein kamen (Abb. 9) [17].

\section{Westliche Anatomie}

Das erste massgebliche westliche Werk über anatomische Forschungern erschien mehr als 500 Jahre nach dem chinesischen Kupfer-Menschen. Es stammt von Andreas Vesalius und wurde als „De Humani Corporis Fabrica“ 1543 in Basel veröffentlicht (Abb. 7) [14].

\section{Folgerungen für Praxis und Forschung der modernen Akupunktur}

Bereits in den 80er Jahren hat HeINE auf die anatomische Fundierung der Akupunktur hingewiesen [18]. Im Rahmen unseres Themas sollen vor allem für die Praxis relevante Fakten herausgestellt werden [19]:

1. Wenn der Arzt die am Unterarm gelegene Struktur Shou San $L i$ 手三里 („Punkt Dickdarm 10“) mit der Nadel (roter Griff) punktiert, wie es die Abbildung 11 zeigt, beeinflusst er damit der modernen Medizin wohlbekannte Körpergewebe, nämlich:

- die Haut und das Unterhautgewebe am seitlichen Unterarm

- den Nervus cutaneus antebrachii medialis (Ramus anterior) (1)

- die Vena mediana antebrachii (3)

- die Arteria radialis mit den zugehörigen Venae radiales (6)

- den Nervus cutaneus antebrachii lateralis (7)

- die Arteria recurrens radialis (8)

- die Vena cephalica (9)

- den oberflächlichen Ast des Nervus radialis (10)

- den Musculus extensor carpi radialis longus (12)

- den Musculus supinator (Zielstruktur des Nadeleinstichs im roten Kreis) (13)

- den tiefen Ast des Nervus radialis (14)

- den Musculus extensor carpi radialis brevis (16)

- den Streckermuskel des Kleinfingers und der Finger (17)
- das Periost des Radius (Zielstruktur des Nadeleinstichs im roten Kreis) (18)

- den Nervus cutaneus antebrachii posterior (19)

- die Vena basilica (32)

sowie alle übrigen in Abbildung 11 verzeichneten Strukturen. Diese anatomischen Strukturen müssen dem Akupunkteur bekannt sein, damit er die Wirkung seines Nadeleinstichs umfassend beurteilen kann, am Patienten keinen Schaden anrichtet und keinen medizinischen Kunstfehler begeht. Dabei ist anzumerken, dass die Einstichtiefe der Nadeln je nach Lebensalter und Konstitution des Patienten erheblich variieren kann. Auch bei flacherem Nadeleinstich werden über Rezeptoren die Hautgefässe (Abb. 11 Ziffern 3, 9, 32) und -nerven (Abb. 11 Ziffern 1, 7, 19) stimuliert, die bekanntlich Ramifikationen tiefer liegender Gefässe und peripherer Nervenverläufe sind. In den Abbildungen ist jeweils die maximale Stichtiefe dargestellt.

In Abbildung 12 ist ein Querschnitt durch das Foramen Shang $J u X u$ 上巨 虚 (im Westen als „Punkt Magen 37“ bezeichnet) an der Vorderseite des Unterschenkels dargestellt; gezeigt werden folgende Körperstrukturen:

- die Haut und das Unterhautgewebe

- das Schienbein (Tibia) (2)

- die Membrana interossea cruris (3)

- der Musculus tibialis anterior (4)

- der Musculus tibialis posterior als Zielstruktur mit der Nadel im roten Kreis (5)

- der lange Streckermuskel der Zehen (6)

- die Arteria tibialis anterior mit den zugehörigen Venen (7)

- der Nervus peronaeus profundus (8)

- das vordere Septum intermusculare des Unterschenkels (9)

- der Nervus peronaeus superficialis (11)

- das hintere Septum intermusculare des Unterschenkels (13)

- das Wadenbein (Fibula) (14)

- der Nervus cutaneus surae lateralis (18)

- die Arteria peronaea und die zugehörigen Venen (23)

- der Nervus tibialis (25) 


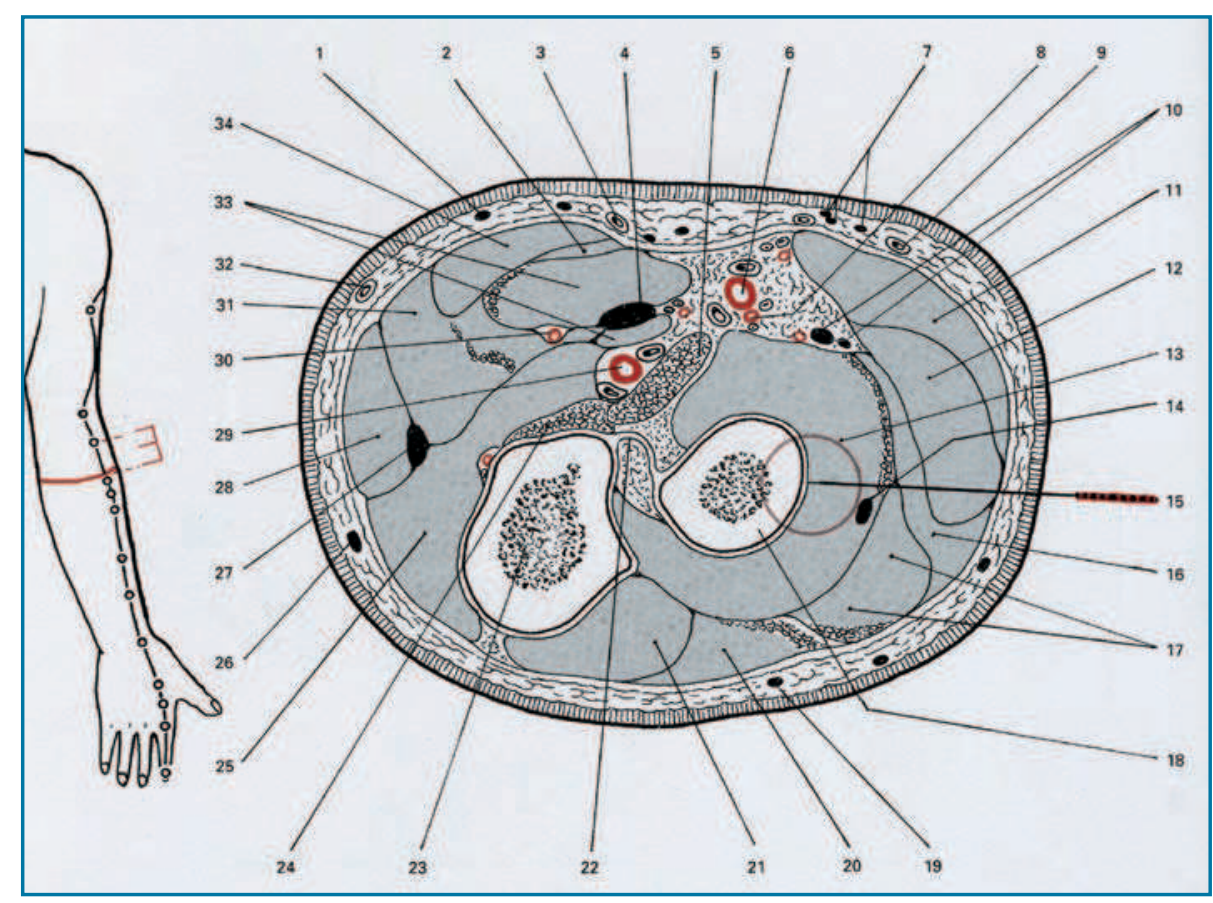

Abb. 11. Anatomischer Querschnitt durch den Unterarm, Struktur Shou San Li 手三里 (im Westen als „Punkt Dickdarm 10" bezeichnet) [19].

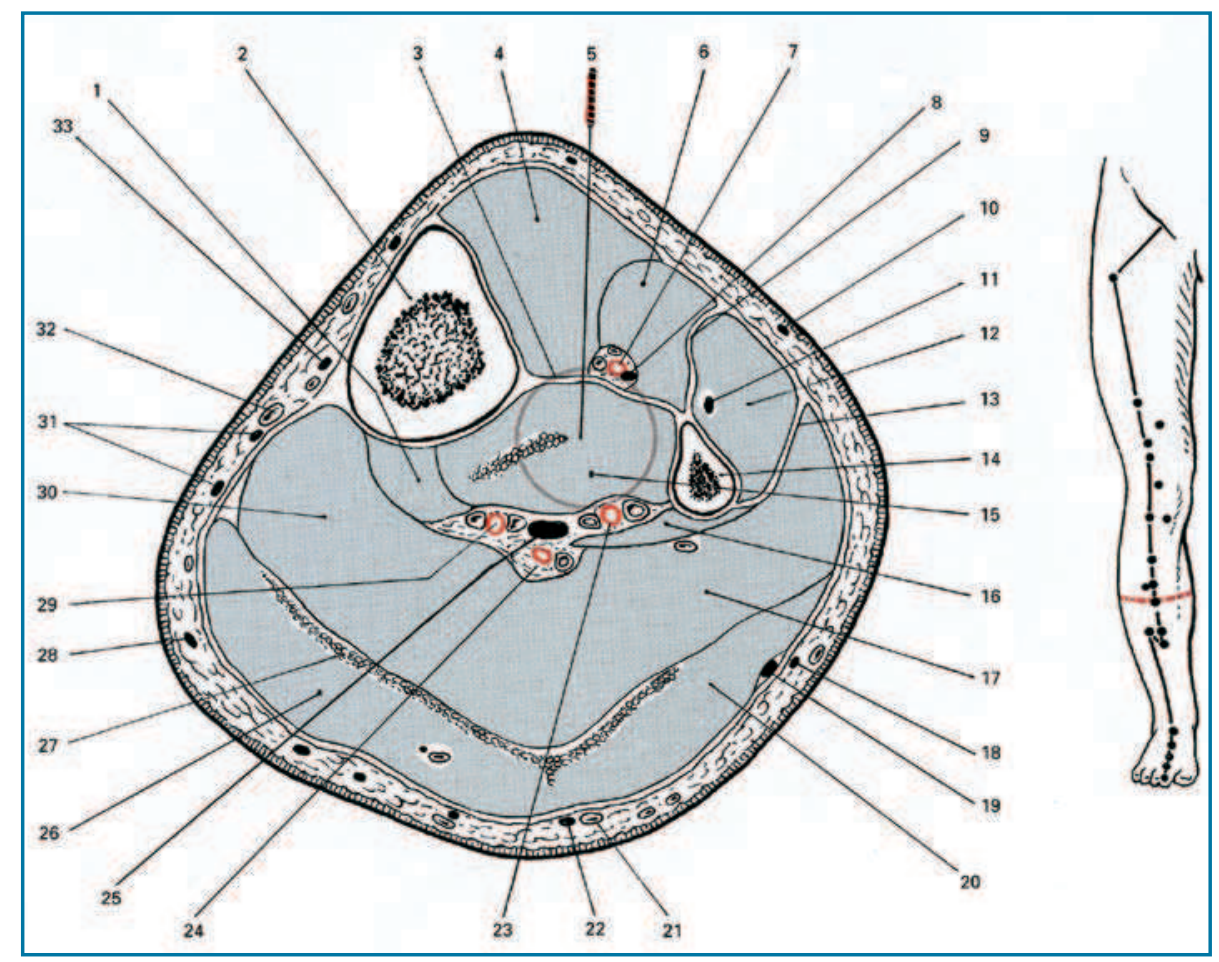

Abb. 12. Anatomischer Querschnitt durch das Foramen Shang Ju Xu 上巨虛 (im Westen „Punkt Magen 37" genannt) an der Vorderseite des Unterschenkels [19].

- die Arteria und die Vena tibialis posterior (29).

Weder „Meridiane“, in denen laut verbreiteter westlicher Auffassung eine imaginäre Energie fliessen soll, noch „Punkte“ sind auf den Abbildungen 11 und 12 zu sehen. Warum? Weil derartige Phantasiegebilde in der chinesischen Medizin nie existiert haben. Es ist bekannt, dass Akupunkteure die hier abgebildeten Strukturen beim anatomisch unkontrollierten Einstich verletzt haben, wodurch Gefässläsionen (Blutungen, Hämatome) und Nervenverletzungen mit Entzündungen oder andauernden Nervenschmerzen im Bereich der Einstichkanäle entstanden sind. Wieso konnte das selbst Ärzten passieren? Weil diese nicht adäquat in Akupunktur ausgebildet waren, weil sie vor allem nichts über die anatomischen Strukturen wussten, mit denen sie es in Wirklichkeit zu tun hatten.

Sämtliche oben aufgeführten Gewebe sind bei der Akupunktur des Foramens Shang-Ju-Xu beteiligt. Der Akupunkteur, einerlei ob Arzt oder Nichtarzt, muss sie deshalb kennen und vor dem Einstich seiner Nadeln sorgfältig einzeln identifizieren. Leider wird diese Grundlage der modernen Nadeltherapie, die überhaupt erst eine zuverlässige Wirkung ermöglicht, gegenwärtig im Westen (aber auch in weiten Teilen Chinas) vernachlässigt. Es ist deshalb nicht verfehlt $\mathrm{zu}$ behaupten, dass die übliche Praxis der Akupunktur mit den historischen Dokumenten und deren wissenschaftlichem Anspruch wenig zu tun hat. Bei den entstandenen Verletzungen musste es sich also um medizinische Kunstfehler handeln.

Beispielhaft zu nennen ist in diesem Zusammenhang die auf Kosten der Krankenversicherten von den deutschen Krankenkassen mit einem Aufwand von Hunderten Millionen Euro durchgeführte Untersuchung zur Wirksamkeit der Akupunktur (so genannte Gerac-Studie) [21,22,23]. Hier wurden die Kontroll- bzw. PlaceboGruppen nicht nach anatomisch-physiologischen Kriterien aufgestellt, was eine viel genauere Unterscheidung der Kollektive ermöglicht hätte, sondern nach der irrtümlichen Annahme von „Meridianen“ und „Punkten“. Unter morphologischen Aspekten hat darum der deutsche Anatom H. Heine die Gerac-Studie kritisiert [24]. Denn aufgrund des oben geschilderten falschen westlichen Denkmodells zur Akupunktur unterschieden die Untersucher die von ihnen so genannten „korrekten Einstich-Punkte" nicht nach anatomisch-physiologischen Kriterien von den angeblichen „Placebo-Punkten“, 


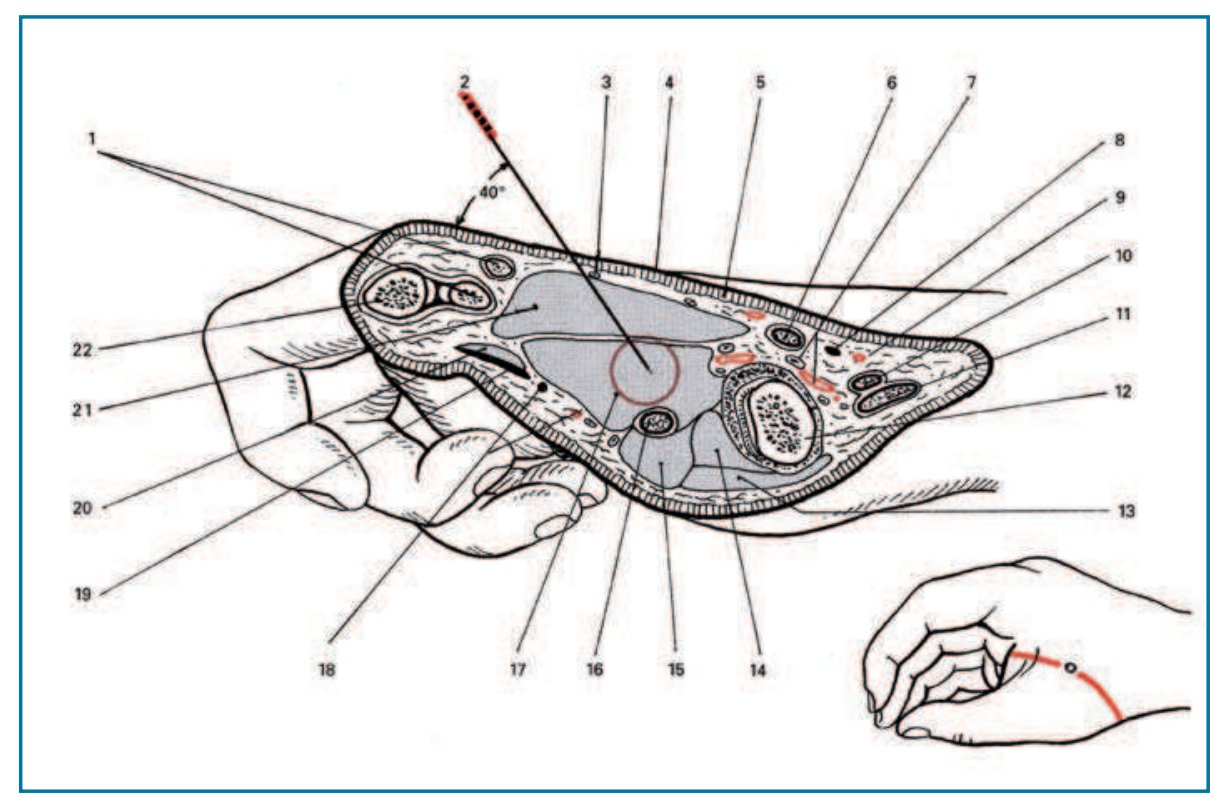

Abb. 13. Anatomischer Längsschnitt durch das Akupunktur-Foramen He-Gu 合谷 [19].

weshalb sich die Gruppe mit der so genannten „echten“ Akupunktur nur unerheblich von der Placebo-Gruppe abhob, was schliesslich zu der von Medien, Schulmedizinern und Ärzteverbänden verbreiteten Unterstellung führte: Jeder Mediziner dürfe bei der Akupunktur beliebig hin stechen wo er wollte. Denn das therapeutische Ergebnis sei ja immer etwa das gleiche (gemäss Gerac-Studie zwischen $48 \%$ und $52 \%)$ [21,22,23]. Solche Ergebnisse, die kaum jemand adäquat zu deuten wusste, wurden zur wissenschaftlichen Farce, weil westlichen Akupunkteuren die im vorliegenden Beitrag erläuterten historischen Zusammenhänge offensichtlich unbekannt sind. Skandalös sind solche fragwürdigen Ergebnisse der Studie nicht zuletzt deshalb, weil daraufhin deutsche Krankenkassen eine mehr schlecht als recht in deutschen Arztpraxen durchgeführte Akupunktur vergüten. Denn so werden die gegen Krankheit versicherten Patienten, die die Gerac-Studie letztlich bezahlt haben, bezüglich der Wirkungsweise der Akupunktur hinters Licht geführt. Über die eigentliche medizinisch-wissenschaftliche Sensation der Gerac-Studie, nämlich den Nachweis, dass eine Kontrollgruppe mit schulmedizinisch Behandelten nur etwas mehr als Hälfte des Therapie-Erfolges im Vergleich mit der Akupunktur (ca.
$28 \%$ ) erbrachte, schwieg man sich bei der Berichterstattung weitgehend aus.

\section{Zur Wirkungsweise der Akupunktur}

Wie wirkt Akupunktur? Die Antwort heisst: über die oben für zwei Akupunktur-Strukturen (Shou-San-Li und Shang-Ju-Xu) exemplarisch dargestellten Muskeln, Nervenbahnen und Gefässverläufe (neuro-vaskuläre Bündel) sowie Sehnenzüge. Wer weiterhin behauptet, die klassische chinesische Medizin habe keine Anatomie gekannt, ist unzureichend informiert. Denn das Gegenteil ist der Fall: Wir können nur staunen über den Umfang und die Qualität des frühen anatomischen Wissens, das uns in klassischen AkupunkturWerken wie dem Buch Huang-Di NeiJing Ling-Shu aber auch in anderen chinesischen Originalen wie dem Text zum Kupfermenschen [16] überliefert wird.

Übrigens geben die meisten originalen chinesischen Namen der Foramina („Punkte“) die Anatomie und Topografie der Akupunkturstrukturen richtig wieder. So heisst die Übersetzung für $H e-G u$ 合谷 (im Westen „Dickdarm 4“ genannt) „Treffpunkt im Tal“ bzw. „Treffpunkt im Knochental“. Das bezieht sich gemäss Abbildung 13 auf die Haut (4) und die Unterhautgewebe (5) sowie auf das Venengeflecht am Handrücken (3), auf den 1. Mittelhandknochen (1), die Muskel-Verbindungen zwischen dem 1. und 2. Mittelhandknochen, d.h. auf den 1. dorsalen Musculus interosseus (21) und auf die Zielstruktur der Nadel (2) im roten Kreis, den Musculus adductor pollicis (17), ferner auf die Arteria digitalis palmaris communis, den Nervus digitalis palmaris communis (18) und die Sehne des langen Daumenbeugemuskels (16) zusätzlich zu allen anderen in dieser Abbildung aufgeführten Strukturen.

Eine in den 90er Jahren am Deutschen Forschungsinstitut für Chinesische Medizin in Freiburg i.Br. durchgeführte Untersuchung zum topographischanatomischen Bezug der originalen chinesischen Namen der AkupunkturForamina („Punkte“), ergab ein überzeugendes Resultat zu Gunsten ihrer topographisch-anatomischen Benennung, was aus den Tabellen 3-5 hervorgeht [19].

\section{Schlussfolgerungen}

Vom wissenschaftlichen Sachverhalt her gesehen ist es unkorrekt, aber gängige westliche Praxis der Akupunktur, nicht die zweifelsfrei vorliegenden anatomischen Strukturen zu verstehen und zu behandeln, sondern lediglich irgendwelche „Punkte“ zu stechen und letztere als Dickdarm 4, Dickdarm 10, Magen 37, Lunge 5, Leber 3 oder Milz 6 auszugeben. Diese westliche Nummerierung mag vielleicht eine mnemotechnische Funktion haben; wissenschaftlich fundiert ist sie nicht. Denn solche Kürzel enthalten keinerlei medizinisch relevante, geschweige denn bei wissenschaftlichen Forschungen verwertbare Informationen. In China sind derartige Abkürzungen unbekannt. Darum muss am Anfang jeder vernünftigen Akupunkturausbildung im Westen das Studium der korrekten anatomischen Nomenklatur der tatsächlichen anatomischen Strukturen einschliesslich der mit ihnen gekoppelten und inneren Organe stehen, mit denen es die Akupunkteure und chinesische Mediziner in der Tat zu tun haben, und die historisch und natürlich dieselben sind 
Tab. 3. Prozentuale Aufschlüsselung der originalen chinesischen Namensgebung für die Akupunktur-Foramina (Jing Xue 經穴) der 14 Haupt-Gefässe („Meridiane“): 72\% der Namen beruhen auf der Anatomie und Topographie der Foramina („Punkte“); $14 \%$ der Namen ergeben sich aus ihrer medizinischen Wirkung; 12\% gehen auf die chinesische Physiologie zurück, 2\% der Namen ergeben sich aus sonstigen Anhaltspunkten [19].

\begin{tabular}{|lccccc|}
\hline Gruppe & Topographie & Wirkung & $\begin{array}{l}\text { Physiologie } \\
\text { (chin. Bedeutung) }\end{array}$ & Sonstige & Summe \\
\hline Lu & 10 & - & - & 1 & 11 \\
Di & 17 & 2 & - & 1 & 20 \\
Ma & 40 & 4 & 1 & - & 45 \\
Mi & 11 & 6 & 3 & 1 & 21 \\
H & 3 & 2 & 4 & - & 9 \\
Dü & 16 & 2 & 1 & - & 19 \\
BI & 51 & 8 & 8 & - & 67 \\
N & 15 & 5 & 6 & 1 & 27 \\
P & 8 & - & 1 & - & 9 \\
BE & 17 & 3 & 3 & - & 23 \\
G & 28 & 9 & 7 & - & 44 \\
Le & 11 & 1 & 2 & - & 14 \\
LG & 20 & 5 & 3 & - & 28 \\
DG & 12 & 4 & 5 & 3 & 361 \\
\hline Summe & 259 & 51 & 44 & 7 & 100 \\
$\%$ & 72 & 14 & 12 & 2 & \\
\hline
\end{tabular}

Tab. 4. Prozentuale Aufschlüsselung der originalen chinesischen Namensgebung für die sogenannten Zusatz-Foramina (Qi Xue 奇穴): 71\% der Namen beruhen auf der Anatomie und Topographie der Zusatz-Foramina („Punkte“); 25\% ihrer Namen ergeben sich aus der medizinischen Wirkung, 1\% gehen zurück auf die traditionelle chinesische Physiologie; $3 \%$ der Zusatz-Foramina wurden nach sonstigen Eigenschaften benannt [19].

\begin{tabular}{|llllll|}
\hline Gruppe & Topographie & Wirkung & $\begin{array}{l}\text { Physiologie } \\
\text { (chin. Med.) }\end{array}$ & Sonstige & Summe \\
\hline KuH & 20 & 13 & 0 & 1 & 34 \\
ThuA & 23 & 12 & 3 & 1 & 39 \\
RÜL & 25 & 10 & 0 & 3 & 38 \\
OE & 40 & 8 & 0 & 1 & 49 \\
UE & 36 & 7 & 0 & 0 & 43 \\
\hline Summe & 144 & 50 & 3 & 6 & 203 \\
$\%$ & 71 & 25 & 1 & 3 & 100 \\
\hline
\end{tabular}

Tab. 5. Prozentuale Aufschlüsselung der originalen chinesischen Namensgebung für die sogenannten Neu-Foramina (Xin Xue): 53\% der Namen beruhen auf der Anatomie und Topographie der Neu-Foramina („Punkte“), 47\% ihrer Namen ergeben sich aus der medizinischen Wirkung, auf die traditionelle chinesische Physiologie oder Sonstiges gehen bei den Neu-Foramina keine Namen zurück [19].

\begin{tabular}{|lccllc|}
\hline Gruppe & Topographie & Wirkung & $\begin{array}{l}\text { Physiologie } \\
\text { (chin. Med.) }\end{array}$ & Sonstige & Summe \\
\hline KuH & 17 & 20 & 0 & 0 & 37 \\
Abd & 3 & 3 & 0 & 0 & 6 \\
RÜL & 6 & 12 & 0 & 0 & 18 \\
OE & 15 & 6 & 0 & 0 & 21 \\
UE & 21 & 15 & 0 & 0 & 36 \\
\hline Summe & 62 & 56 & 0 & 0 & 118 \\
$\%$ & 53 & 47 & 0 & 0 & 100 \\
\hline
\end{tabular}

wie die Strukturen, Bahnen und Körpergewebe der modernen westlichen Heilkunde.

\section{Literatur}

1. Huang-Di Nei-Jing Ling-Shu, 黃帝內經靈樞 Originalausgabe, Wuhan/China, 1852 und moderne Ausgaben.

2. Han Annalen Han Shu 漢書, Biografie des
Wang Mang, zit. nach K. Chimin Wong and Wu Lien-Teh, History of Chinese Medicine Being a Chronicle of Medical Happenings in China from Ancient Times to the Present Period, 2nd ed, National Quarantine Service, Shanghai 1936, S. $194 \mathrm{ff}$.

3. Huang-Di Nei-Jing Su-Wen, 黃帝內經素問, Originalausgabe, Soochow/China, 1892 (und moderne Ausgaben).

4. Chimin Wong K. and Wu Lien-Teh, History of Chinese Medicine, op. cit., S. 196.
5. Teizo Ogawa, History of Medicine, 1964 (japanisch).

6. Zhonghua Yishi Zazhi, 中華醫史雜誌, Chinese Journal of Medical History, Beijing 1994;24(2):68-74.

7. Teizo Ogawa, The Beginnings of Anatomy in Japan, Okajimas Fol. anat. jap. 1975;52: 59-72.

8. Huang-Di Nei-Jing Ling-Shu, 黃帝內經靈樞, op. cit., Kap. 31

9. Huang-Di Nei-Jing Ling-Shu, 黃帝內經靈樞, op. cit., Kap. 12

10. Feneis H, Dauber W, Tu-Jie Ren-Ti Jie-Pou-Xue Shou-Ce 圖解人體解剖學手冊, Handbook of Human Anatomy, in Chinesisch, Thieme/HoChi, Stuttgart-New York-Taipei, 2001.

11. Ren-Ti Jie-Po Xue，人体解剖學，Lehrbuch der Anatomie des menschlichen Körpers, Hygiene Verlag der Volksrepublik China, Beijing 1977-1978.

12. Huang-Di Nei-Jing Ling-Shu, 黃帝內經靈樞, op. cit., Kap. 10.

13. Kendall D, DAO of Chinese Medicine, Understanding an Ancient Healing Art, Oxford University Press, Hong Kong-New York, 2002.

14. Vesalius A, De Humani Corporis Fabrica, Basel 1541

15. Hongchien $\mathrm{Ha}$, A Survey on Bronze Statue of Acupuncture, China Medical College Annual Bulletin 1988;14.

16. Tong-Ren Zhen-Jiu-Shu-Xue-Tu-Jing, Der klassische Text zu den Illustrationen der Foramina für die Akupunktur und Moxibustion auf dem Kupfermenschen, von Wang Wei-Yi (987-1067), um 1027 in der Nördlichen SongDynastie (960-1126). Enthalten in: ZhongCuo Yi-Xue Da-Cheng (Das Grosse Werk der Chinesischen Heilkunde), Shanghai Da-Dong Shu-Ju (Shanghai Buchladen des Grossen Ostens), 1936-1937, Shanghai.

17. Hongchien $\mathrm{Ha}$, persönliche Mitteilungen.

18. Heine $H$, Anatomische Struktur der Akupunkturpunkte, Deutsche Zeitschrift für Akupunktur 1988;2:26-30.

19. Schnorrenberger C, Compendium Anatomicum Acupuncturae, Lehrbuch und Atlas anatomischer Akupunktur-Strukturen (unter Mitarbeit von Hongchien Ha), Walter de Gruyter Verlag, Berlin-New York 1996.

20. Schnorrenberger C, Morphological Foundations of Acupuncture, an Anatomical Nomenclature of Acupuncture Structures; Acupuncture in Medicine, Journal of the British Medical Acupuncture Society1996;14(2):89-103.

21. WeidenhammerWetal., Das Modellvorhaben Akupunktur der Ersatzkassen, Deutsche Zeitschrift für Akupunktur 2002;3:183-192.

22. Brinkhaus B et al., Acupuncture Randomized Trials (ART) in Patients with Low Back Pain and Osteoarthritis of the Knee, Forschende Komplementärmedizin und klassische Naturheilkunde 2003;10:165-191.

23. Neue Zürcher Zeitung, Akupunktur sticht Schulmedizin aus, in: Forschung und Technik, NZZ Nr. 257, 3.11.2004, S. 59.

24. Heine $H$, Akupunktur - Quo vadis? Dtsch Med Wochenschrift 2006;131:1552-1553.

\section{Korrespondenzadresse:}

Prof. h.c. (China Medical University, Taichung, Taiwan, Republic of China) Dr. med. Claus C. Schnorrenberger Lifu International College of Chinese Medicine Karl Jaspers-Allee 8, CH-4052 Basel e-mail: lifu@gmx.ch nwww.lifu-college.ch 